\title{
A CYBERNETIC PERSPECTIVE ON CAR FOLLOWING IN FOG
}

\author{
Erwin R. Boer, ${ }^{1}$ Stéphane Caro, ${ }^{2}$ Viola Cavallo ${ }^{2}$ \\ ${ }^{1}$ LUEBEC \\ San Diego, California, USA \\ E-mail: ErwinBoer@LUEBEC.com \\ ${ }^{2}$ INRETS - LPC \\ Arcueil, France
}

\begin{abstract}
Summary: Drivers often drive at a closer time headway (THW) in fog than in clear whether conditions for similar speed ranges (White \& Jeffery, 1980). Closer following is generally considered more dangerous. The hypothesis pursued in this paper is that drivers experience a perceptual-motor benefit from driving closer in fog that results in greater (or equivalent) safety and reduced driving demand. A computational car following model with an experimentally constructed perceptual module is introduced and used to demonstrate that under some conditions, closer following in fog is indeed beneficial because it effectively reduces drivers' perceptual delay by a sufficient amount to improve controllability of the gap so much that the variability in THW reduces more than (or as much as) the adopted decrease in target THW.
\end{abstract}

\section{CAR FOLLOWING IN FOG}

The two cardinal components of car following are perception and control of gap changes. When one or both are affected by the lead vehicle speed characteristics, the weather condition, the light conditions, or the following vehicle dynamics, drivers adopt a different car following control strategy (Boer, Ward, Manser, \& Kuge, 2006). This means that drivers adopt different target THWs and different response characteristics to perceived changes in THW. In other words, the driver changes his goals, response delay (attention), and control gains to achieve his desired safety margin (e.g., minimum THW).

In fog, perception of gap changes is deteriorated (see companion paper Caro, Cavallo, Boer, \& Vienne (2007) for references). It takes drivers longer to detect a gap change in fog than in clear weather conditions. This means that the delay between actual change in gap and response to that change in gap is increased. This increase in delay results in greater variability in THW, and therefore a shorter minimum THW. Under clear weather conditions, drivers can increase their target THW to achieve their desired safety margin. However, under fog conditions, an increase in target THW increases their perceptual delay more than under clear conditions. If their delay increases so much that the resulting increase in THW variability is greater than the increase in the adopted target THW, then the net effect is a shorter minimum THW rather than the desired longer minimum THW. If this is true, then the converse is also true, namely, that a shorter target THW may reduce the perceptual delay enough to cause the variability in THW to reduce by more than the reduction in target THW, thus yielding a higher minimum THW than was obtained at the longer THW. It should be noted that different target THWs also require different control gains (Boer, et al., 2006). The model presented here demonstrates this effect. 


\section{DRIVER MODEL OF CAR FOLLOWING IN FOG}

The car following model structure presented in Kuge, Boer, Yamamura, Ward, \& Manser (2006) is used as the basis for the model presented herein. Two changes to the model were needed. The first is a change in the control input variables from distance and relative velocity to visual angle and visual angle rate. The particular visual angle used in the present model is the one that subtends the width of the vehicle. Integration of multiple cues is ignored. The second change is to add a perceptual module that represents the intermittency and delayed perception caused by just noticeable differences (JNDs) in relevant visual cues (e.g., visual angle), plus the fact that these JNDs are higher in fog than under clear conditions. Many factors are ignored but the resulting model suffices to demonstrate the fact that closer following under some conditions yields a perceptual-motor benefit and therefore a reduced driving demand and increased safety.
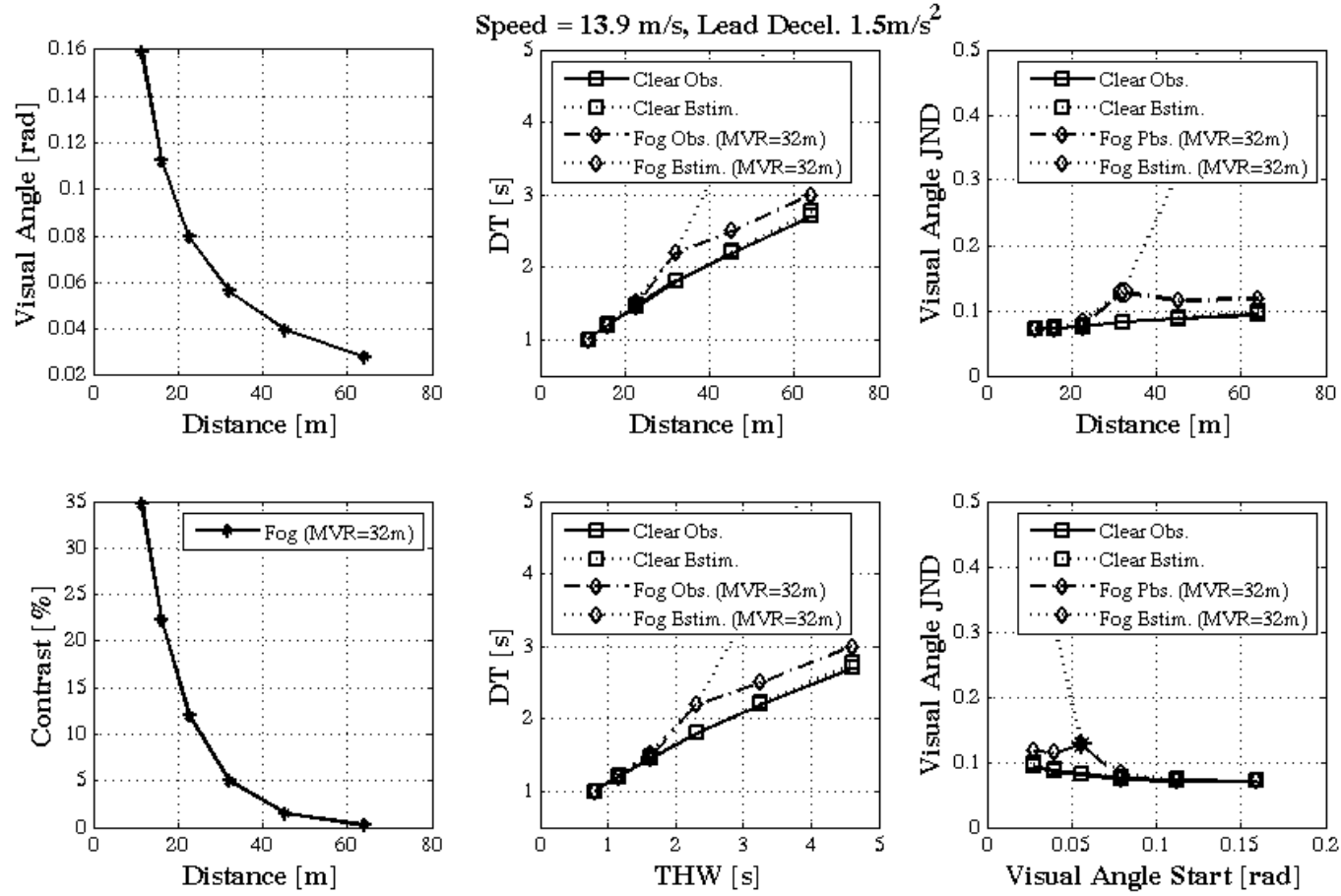

Figure 1. Experimental observations from Caro, et al., (2007). Relationships between distance, visual angle and contrast (left panels). Detection times as a function of dist. and THW (middle panels). JND in vis. ang. space as a function of vis. ang. and dist. in clear and fog conditions (right panels). The parameterized JND for fog and clear conditions as a function of vis. ang. are shown as dotted lines. For the JND fit under fog conditions, only observed JNDs at vis. angles greater than 0.05 rad were used (see text for details)

\section{Perceptual Model}

An important component responsible for the difficulties encountered while driving in fog is that the JND for detection of changes in relevant perceptual cues increases. To model this effect, we used the experimental data from our companion paper (Caro, et al., 2007) to establish a relationship between visual angle (angle subtended by the width of the $1.8 \mathrm{~m}$-wide vehicle) and JND in visual angle change. A clear and a fog condition with a Meteorological Visibility Range 
(MVR) of $32 \mathrm{~m}$ are used. For the clear condition, the contrast was always 100\% (i.e., (luminance of lead vehicle luminance minus luminance background)/(luminance background)). The experiment measured detection times in clear and fog at gap distances of $11.3,16,22.6,32,45.3$, and $64 \mathrm{~m}$ at $50 \mathrm{kph}\left(13.9 \mathrm{~m} / \mathrm{s}\right.$ ) when the lead vehicle decelerated at $1.5 \mathrm{~m} / \mathrm{s}^{2}$ (see Caro, et al., 2007 for details) For the fog condition, these distances corresponded to contrasts of 34.7, 22.3, 12, 5, 1.5 , and $0.25 \%$, respectively. Using simple conversion formulas, the relationship between visual angle at the onset of deceleration and JND can be computed from these detection time observations for both conditions. To parameterize the fog condition, we only used the detection times up to the distance where the outline was visible (i.e., $5 \%$ contrast at a distance of $32 \mathrm{~m}$ ) because at the moment, the model only incorporates the effect of contrast changes, not drop out of perceptual cues on car following behavior or the effect of fog lights. Figure 1 shows the various relationships including the parameterized fits (equations shown in set of model equations shown below) we created for the relationship between visual angle and JND for the clear and the fog condition.

The perceptual component of the model is comprised of a "filter" that simply waits until the visual angle has changed beyond the JND (Boer, 1999). The JND threshold depends on the visual angle, as well as the weather condition. Each time JND is exceeded, a new perception / observation is made that is used to update an internal representation of the visual angle (i.e., the one used for control). At the moment, the model simply replaces the internal representation with the correct one. In actuality, each perception has some uncertainty, which causes the internal representation to update cautiously, but that mechanism is ignored in the current simulations.

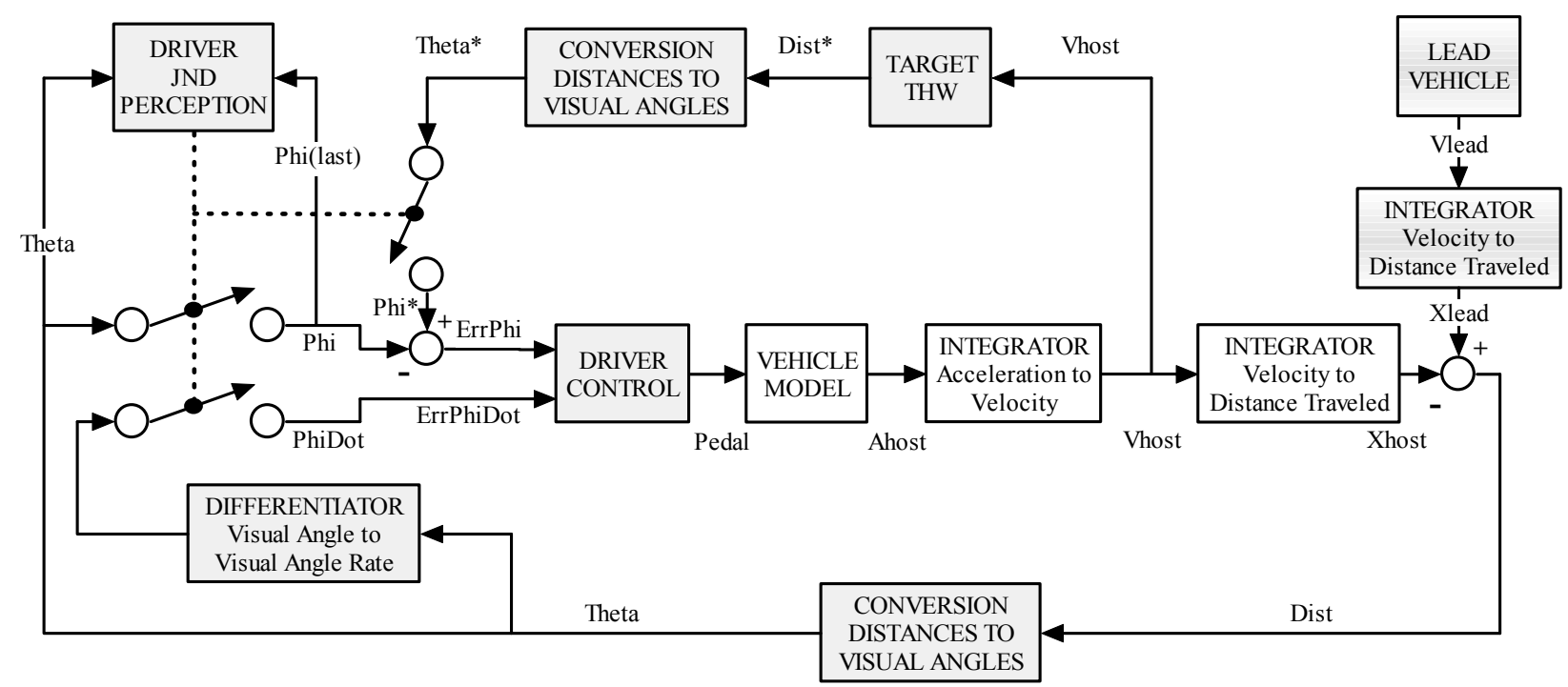

Figure 2. Driver model of car following under restricted visibility conditions. The "JND Perception" box represents a switch that results in a new observation of a changed visual angle when the visual angle has changed relatively by more than JND. JND is not constant as seen in Figure 1, but a function of the visual angle "observed" at the last time when JND was exceeded (i.e., Phi(last)). Driver control inputs are visual angle and visual angle rate (unlike the distance and relative velocity used in most existing models) Gap Change JND Model 
A new driver car following control model shown in Figure 2 was developed that uses visual angle and visual angle rate as control input to the driver (perceptually more plausible than often used distance and relative velocity). The model is purposefully held simple so that the effects of following at different target THWs under clear and foggy conditions can be understood easily. Due to space constraints, the block diagram and the set of equations are meant to speak for themselves. Any necessary additional detail is given below, adjacent to the equations.

At each time step (update time-interval $\mathrm{Ts}=0.05 \mathrm{~s}$ ) the following set of equations are executed:

$\operatorname{Dist}(n)=X_{\text {Lead }}(n-1)-X_{\text {Host }}(n-1)$

$\operatorname{Dist}^{*}(n)=V_{\text {Host }}(n-1) \times T H W^{*}$

$\left\{T H W^{*}\right.$ is the Target THW. $\}$

$\operatorname{ErrDist}(n)=\operatorname{Dist}^{*}(n)-\operatorname{Dist}(n)$

\{These distance and velocity errors are not used

$\operatorname{ErrVel}(n)=V_{\text {Lead }}(n-1)-V_{\text {Host }}(n-1)$

for control but only for plotting in Fig. 4.\}

$\operatorname{Theta}(n)=2 \arctan \left(\frac{w}{2 \operatorname{Dist}(n)}\right)$

\{The width of the lead vehicle is $\mathrm{w}=1.8 \mathrm{~m}$. \}

$\operatorname{Theta}^{*}(n)=2 \arctan \left(\frac{w}{2 \operatorname{Dist}^{*}(n)}\right)$

ThetaDot $(n)=$ Theta $(n)-$ Theta $(n-1) /$ Ts

$J N D_{\text {Clear }}=0.065+\frac{0.000979}{\text { Phi (last })+0.001}$

\{See experimental results Figure. 1 for shape

$J N D_{\text {Fog }}=0.07+\frac{\exp ^{-14.86}}{(\text { Phi }(\text { last }))^{4.17}}$

of the functions and their fit to observations.

When

absolute $\left(\frac{\text { Theta }(n)-\text { Phi }(\text { last })}{P h i(\text { last })}\right) \geq J N D_{\text {Clear or Fog }}$

a new "observation" is made, and the internal Angle and AngleDot representations are updated:

$\operatorname{Phi}($ new $)=\operatorname{Theta}(n-K)$

$\{$ The internal angle representations are denoted

$\operatorname{Phi}^{*}($ new $)=$ Theta $^{*}(n-K)$

by Phi; the true visual angles by Theta.\}

$\operatorname{ErrPhi}($ new $)=\operatorname{Phi}^{*}($ new $)-\operatorname{Phi}($ new $)$

$\operatorname{PhiDot}($ new $)=$ ThetaDot $(n-K)$

$\{\mathrm{K}$ represents a delay of $300 \mathrm{~ms}(\mathrm{~K}=0.3 / \mathrm{Ts}=6)$,

ErrPhiDot $($ new $)=\operatorname{PhiDot}($ new $)$ the inherent lag in the driver and the vehicle.

Phi (last $)=\operatorname{Phi}($ new $)$

\{This is the internal rep. used for JND calcs.\}

Control is based on these internal representations and held constant until a new "observation" is made (i.e. when the visual angle Theta changes more than JND relative to the last internal representation Phi(last)).

$\operatorname{Pedal}(n)=c_{0} \times \operatorname{ErrPhi}($ new $)+c_{1} \times \operatorname{ErrPhiDot}($ new $)$

$A_{\text {Host }}(n)=\operatorname{Pedal}(n) / T s$

$\{$ For demonstration purposes the vehicle

$V_{\text {Host }}(n)=V_{\text {Host }}(n-1)+A_{\text {Host }}(n) \times T s$

"dynamics" model is made purely kinematic.\}

$X_{\text {Host }}(n)=X_{\text {Host }}(n-1)+V_{\text {Host }}(n) \times T s$

$T H W(n)=\left(X_{\text {Lead }}(n)-X_{\text {Host }}(n)\right) / V_{\text {Host }}(n)$ 

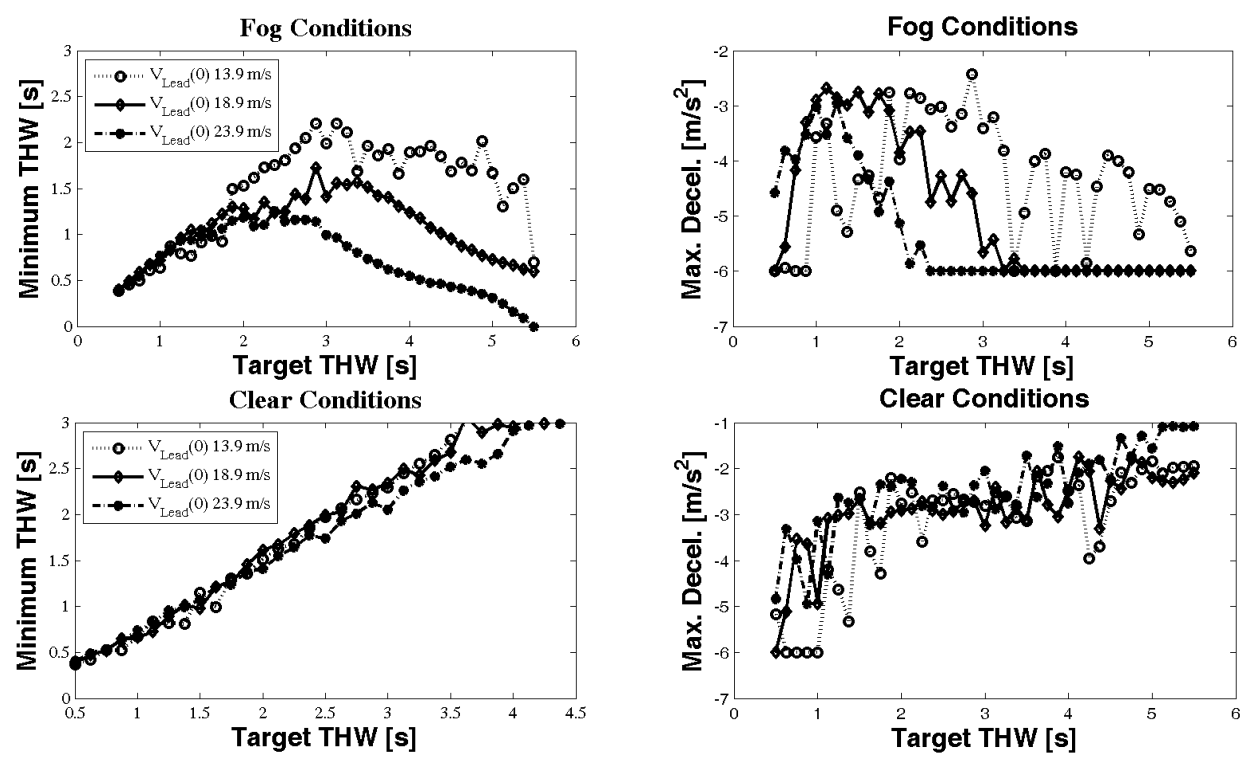

Figure 3. Model run results under fog (top panels) and clear (bottom panels) conditions at different lead vehicle speeds (i.e., 13.9, 18.9, and $23.9 \mathrm{~m} / \mathrm{s}$ ) with different target THWs (0.5 to 5.5s). For each combination, the optimal control coefficients c0 and c1 were identified by searching for those values that yield the smallest distance variance (i.e., assume similar driver vigilance for all runs). For each combination, a model run was simulated using the optimal coefficients; the minimum THW reached (left panels) and the maximum deceleration observed (right panels) were recorded

\section{Model Simulation Results}

The model was run for foggy and clear conditions for a wide range of lead vehicle speeds and target THWs. The lead vehicle speed was kept constant for about 20s, after which it fluctuated sinusoidally with an amplitude of $5 \mathrm{~m} / \mathrm{s}$ and a period set such that the maximum deceleration was $1.5 \mathrm{~m} / \mathrm{s}^{2}$ (i.e., the deceleration magnitude condition used in the Caro, et al., 2007 paper). An example lead vehicle speed profile can be seen in Figure 4 for an initial speed of $13.9 \mathrm{~m} / \mathrm{s}$. The initial conditions for the host vehicle, such as speed and distance to the lead vehicle, were set such that the initial error were both zero (i.e., at target THW with zero relative velocity). Given that drivers optimize their control gains to the conditions at hand (i.e., lead vehicle speed, target THW, expected lead vehicle speed fluctuations), the optimal control gains $\mathrm{c}_{0}$ and $\mathrm{c}_{1}$ were obtained for each combination of lead vehicle speed and target THW separately (see Figure 3 for details). Min. THW experienced was used as a means to determine whether closer following would be safer.

The results shown in the upper left panel in Fig. 3 show clearly that an optimum exists at each of the three simulated speeds; e.g., following at a target THW of $3.25 \mathrm{~s}$ at $13.9 \mathrm{~m} / \mathrm{s}$ results in a longer (i.e., safer) minimum THW than following with a longer target THW. This optimum shifts to lower target THWs when lead vehicle speed increases. It appears that the distance associated with the optimum target THW at each speed is constant at about $50 \mathrm{~m}$, which is where the increase in JND is such that an increase in target THW results in an increase in detection time 
(DT in Figure 1) that yields an increase in THW variability that is greater than the adopted increase in target THW.

Fog Model Run: Near THW=3.25s
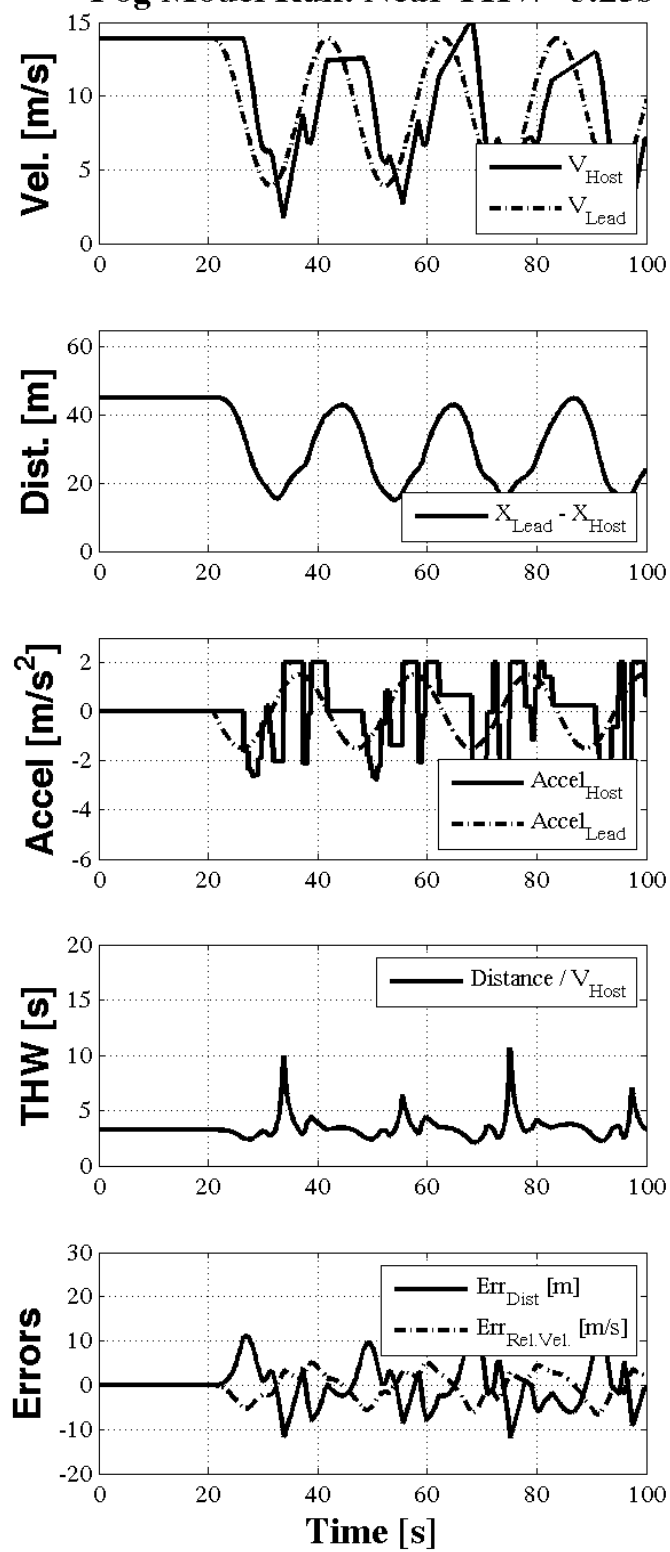

Fog Model Rum: Far THW=4.25s
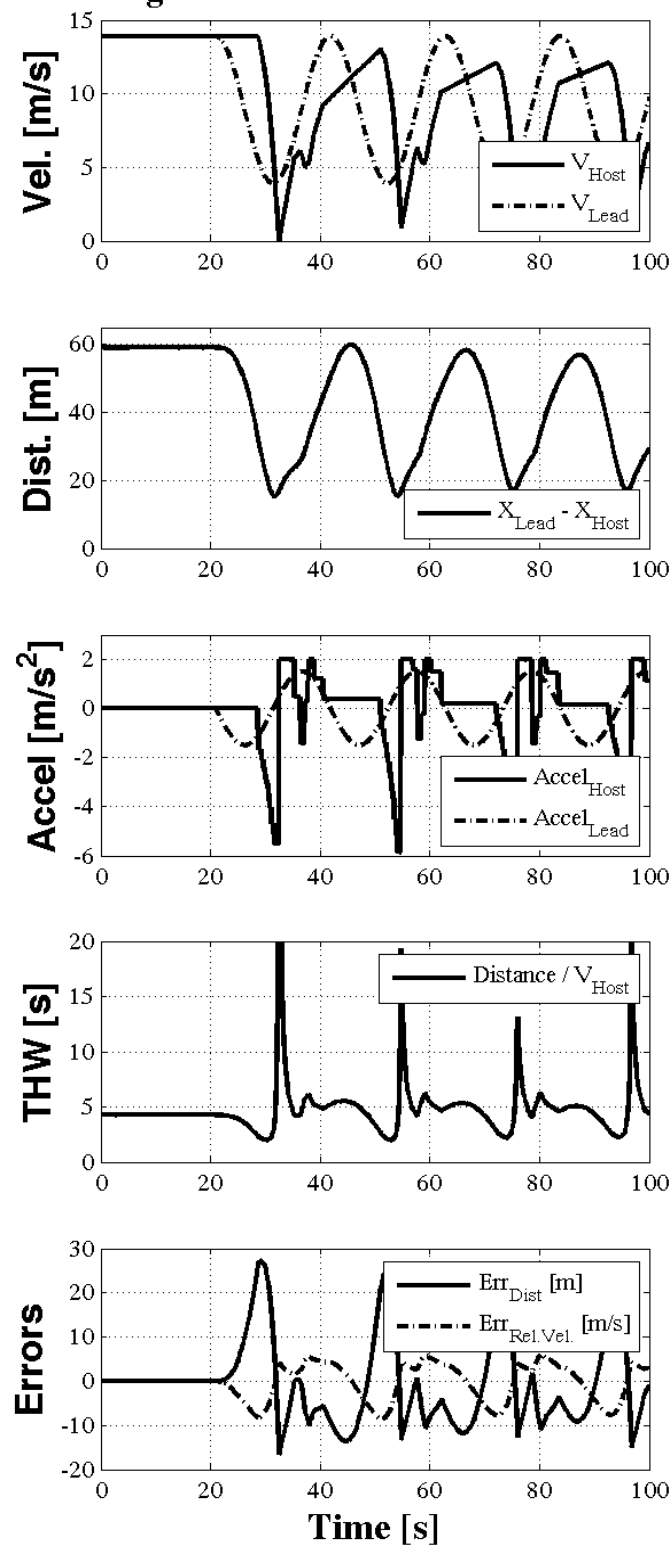

Figure 4. Model simulation runs under fog conditions for a lead vehicle speed of $13.9 \mathrm{~m} / \mathrm{s}$ at the optimum target THW (i.e., left panel at target $\mathrm{THW}=3.25 \mathrm{~s}$ ) and $1.0 \mathrm{~s}$ beyond the optimum target THW (i.e., right panel at target $\mathrm{THW}=4.25 \mathrm{~s}$ ). It is clear that closer following results in smaller errors (bottom panel 5), longer minimum THW (panel 4), less strong decelerations (panel 3), higher frequency control (panel 3), smaller distance fluctuations (panel 2), and higher min. speeds (panel 1). The blocky nature of accelerations is caused by the JND module; not-shown car following under clear conditions does not exhibit such a blocky control due to the fact that JND is exceeded more readily under clear conditions and thus more new observations are available for more continuous control. 
These results clearly show that fog can cause conditions under which closer following is not only safer but also less effortful, as indicated by the fact that the maximum deceleration also intensifies at target THWs longer that the optimum (as shown in the right panels in Figure 3 ).

To demonstrate the effect of closer following in fog, time series of relevant vehicle and driver control variables are shown in Figure 4. The main effect of closer following in fog when the target THW is beyond the optimum (upper two panels Figure 3 ) is the fact that the overall delay in the control loop decreases. Under normal visibility conditions, closer following requires a decrease in delay time if the goal is to maintain the same minimum THW. If the driver can not decrease his perceptual motor delay time any further, the minimum THW will decrease (bottom panels Figure 3). Under foggy conditions, closer following reduces the perceptual delay when the target THW is beyond an optimum. It should be noted that, for example, a reduction in target THW of $100 \mathrm{~ms}$ requires a reduction in delay time much more than $100 \mathrm{~ms}$. This also suggests that fog with different optical characteristics (e.g., due to droplet size caused by different size and density of nuclei in the atmosphere) may show the benefits of closer following at shorter distances.

\section{CONCLUSION}

A computational driver model of car following is presented that uses delayed visual angle and angle rate as control input together with an experimentally constructed perceptual module that uses a visual-angle dependent JND module as a model of when drivers receive new observations for control. The model clearly demonstrates that closer following in fog can under certain conditions lead to safer and less effortful driving. The model does not include the full set of perceptual cues that guide car following in fog, and because of that presents the effect of one perceptual component on car following in fog, namely that of a decreasing contrast on the visual angle subtended by a lead vehicle; ignoring, for example, effects of losing visual cues or the effects of fog lights. The authors recognize that much more work is needed but that these initial results offer an encouraging step towards lifting the fog that currently veils our fundamental understanding of the intricate complexities of car following in fog.

\section{REFERENCES}

Boer, E.R. (1999). A statistical model of looming detection. In: A. G. Gale (Eds), Vision in vehicles VII. Marseille, France, September.

Boer, E.R., Ward, N.J., Manser, M.P., \& Kuge, N. (2006). Driver-model-based assessment of behavioral adaptation. Transactions of SAE Japan, 37(4), July.

Caro, S., Cavallo, V.C., Boer, E.R., \& Vienne, F. (2007). The Influence of Fog on Motion Discrimination Thresholds in Car Following. Proceedings of the 4th International Symposium on Human Factors in Driver Assessment, Training, and Vehicle Design. Stevenson, Washington, USA (July 9-12).

Kuge, N., Boer, E.R., Yamamura, T., Ward, N.J. \& Manser, M.P. (2006). Study on driver's car following abilities based on an active haptic support function. In Proceedings of the $S A E$ 2006 World Congress, SAE 06AE-168, Detroit, Michigan, April 2-6.

White, M.E., \& Jeffery, D.J. (1980). Some aspects of motorway traffic behaviour in fog (TRRL Laboratory Report 958). Crowthorne, UK: Transport and Road Research Laboratory. 